\title{
Faut-il donner un sens philosophique au mot
}

humanisme?

\section{JACQUES \\ CHOMARAT}

Résumé: Du quatorzième au seizième siècle, l'humanisme est souvent pris pour une doctrine qui tend à substituer l'homme à Dieu comme centre du monde. Mais Pic de la Mirandole se borne à affirmer le libre-arbitre de l'homme: il se rattache à la scolastique. De purs humanistes, tels que Pétrarque, Valla, Érasme, reprochent aux scolastiques d'être disciples d'Aristote plus que du Christ, et d'ignorer l'éloquence, indispensable à la prédication. La nouveauté religieuse de l'humanisme est de dire que des païens ont pu être sauvés, ayant vécu selon une morale chrétienne de fait (Socrate) ou l'ayant exprimée dans certaines de leurs oeuvres (Cicéron, Horace, etc.).

$\mathrm{L}$

a critique littéraire et l'histoire des idées font usage de maints concepts flous et flottants: "humanisme" et "humaniste" sont de ceux-là. ${ }^{1}$ Dans sa brochure L'humanisme en Europe au temps de la Renaissance, Jean-Claude Margolin parle très bien de la "polysémie," de la "plasticité," de ce vocable, de son "obscurité"; il en propose néanmoins cette définition: "mouvement intellectuel et culturel caractéristique de la Renaissance, qui a ouvert la voie à une transformation de la vision du monde, [. . . ] à une image nouvelle de l'homme" (p. 8). Il rappelle ensuite qu'umanista dans le jargon scolaire en Italie désignait, dès le quatorzième siècle, le professeur d'humanae litterae ${ }^{3}$ (ou bonae litterae, politiores litterae, etc.): “L'umanista, c'était le professeur de grammaire et de rhétorique, comme l'artista était le professeur "ès arts libéraux" ou le jurista était le professeur de droit"; 2 mais, grammaire et rhétorique étant deux des arts libéraux enseignés par l'artista, qu'est-ce qui 
distinguait l'umanista de l'artista? Quelle était la nouveauté des humanistes? En fait, l' umanista n'enseignait pas méthodiquement grammaire et rhétorique; la première au moins était supposée déjà connue de ses auditeurs. Il expliquait et commentait en détail les oeuvres d'auteurs anciens, poètes, orateurs, historiens, Virgile, Cicéron, etc., alors que l'artista faisait des cours théoriques de grammaire et de rhétorique sans commenter d'oeuvres. De plus l'umanista essayait de parler et d'écrire dans un latin semblable à celui des grands auteurs qu'il commentait; il pratiquait souvent les mêmes genres: poèmes, dialogues, discours, etc. Pour Margolin, la nouveauté des humanistes résidait dans le but qu'ils donnaient à leur enseignement: “ces 'humanités' représentaient un ensemble de disciplines dont la base était constituée par la grammaire, la rhétorique, le commentaire des auteurs (poètes et prosateurs) et dont la finalité propre était de permettre aux jeunes gens d'acquérir leur humanitas, c'est-àdire de devenir des hommes au sens plein du terme, en combinant étroitement un idéal de connaissance et un idéal d'action" (p.9); l'enseignement des lettres aurait donc eu une visée qu' on peut dire philosophique.

Mais en quoi un humaniste qui explique des poèmes de Catulle ou de $\mathrm{Martial}^{3}$ peut-il répondre à cette définition, quel idéal de connaissance et d'action prétendrait-il communiquer? Margolin essaie de le préciser en rappelant d'abord que certains opposent l'humanisme comme une "philosophie de l'homme, centre et maître du monde, à une conception religieuse - et proprement chrétienne - de l'univers, théocentriste, où l'homme, créé à l'image de Dieu, n'occuperait que la première place dans la hiérarchie des créatures" (p. 10). Humanisme serait alors un synonyme discret d'athéisme, car on ne peut pas à la fois faire de l'homme "le centre et maître du monde" et affirmer un Dieu tout-puissant. Cette thèse extrême n'est pas reprise à son compte par Margolin qui rappelle l'existence de ce qu' on a appelé "humanisme chrétien." Mais n'est-ce pas malgré tout une idée voisine qui sous-tend la suite de son analyse, qui donne un sens à sa formule "une image nouvelle de l'homme" et fait de l'expression "l'humanité de l'homme" autre chose qu' une lapalissade? La nouveauté serait que l'homme est en quelque sorte indépendant de Dieu; même s'il lui doit l'existence, c'est lui-même qui se donne son essence, il est ainsi son propre créateur.

Une telle définition philosophique de l'humanisme est-elle fondée? Plus précisément: s'il est tout à fait légitime d'appeler "humanisme" le travail des humanistae, l'étude des lettres antiques, s'il est non moins légitime d'autre part de baptiser "humanisme" une philosophie qui substitue l' homme à Dieu sinon comme centre et maître de l'univers, du moins comme créateur de sa propre 
humanité, est-il permis de jouer sur cette ambiguïté du terme, de glisser du sens $\mathrm{n}^{0} 1$ au sens $\mathrm{n}^{\circ} 2$, de faire comme si, du quatorzième au seizième siècle, les professeurs de lettres étaient ipso facto porteurs ou annonciateurs d'une philosophie nouvelle, et d'une philosophie qui tendrait à remplacer Dieu par l'homme? Pour en avoir le droit il faut une démonstration et non une affirmation a priori. Il y a de plus un troisième sens sur lequel on joue aussi parfois, celui du latin humanitas "bienveillance, bonté, humanité"; ${ }^{4}$ ce sens est évidemment sans rapport nécessaire avec les deux précédents: on peut être serviable et bienfaisant envers son prochain sans être athée et même sans avoir fait ses humanités. Et réciproquement.

$* * *$

Quels arguments donc permettent de donner à humanisme ce sens philosophique? Chez quel auteur peut-on montrer un lien étroit entre amour des lettres antiques et expression d'une philosophie qui affirme de façon nouvelle l" "humanité de l'homme," sa "dignité," sa "liberté"? "Rien ne sert de multiplier les citations, dit Margolin, on les rencontre au détour de chaque paragraphe de nos auteurs [à savoir: Pic, Érasme, Scève, Lefèvre de la Boderie, Paracelse, Bruno, et même Vinci et Michel-Ange], dans leur correspondance, dans leurs poèmes ou dans leur argumentation rhétorique" (p. 13). Magnifique hyperbole, mais une hyperbole n'a jamais été une démonstration: on préférerait tout de même deux ou trois de ces citations, ou même une seule. Margolin se borne à renvoyer à Pic de la Mirandole (1463-1494) dans les termes suivants: "Humanité de l'homme, inséparable de sa dignité, thème constamment repris par les humanistes. On peut considérer le célèbre discours de Pic de la Mirandole De hominis dignitate [. . . comme la proclamation urbi et orbi de l'avènement d'un monde nouveau, où l'homme prend conscience de son éminente fonction" (p.13). Bien que cette éminente fonction ne soit pas définie (sans doute être le créateur de sa propre essence?), on doit voir ici l'argument central de la conception qui fait de l'humanisme, étude des écrivains antiques, la base d'une philosophie nouvelle donnant à l'homme une grandeur jusqu' alors inconnue.

Examinons donc un passage fort connu de cette oeuvre de Pic de la Mirandole, de cette sorte d'encyclique (urbi et orbi) de la nouvelle doctrine (1486). Dieu (eh oui!) s'adresse au premier homme:

Ô Adam, nous ne t'avons donné ni demeure déterminée ni figure propre ni aucun don particulier, afin que tu aies et possèdes selon ton voeu et ta 
préférence la place, la figure, les dons que tu souhaiteras toi-même. Pour tous les autres êtres leur nature bien définie est contenue à l'intérieur de lois prescrites par nous. Toi, sans être contenu par aucune limitation, tu définiras ta nature selon ta volonté au pouvoir de laquelle je t'ai mis. Je t'ai placé au milieu du monde afin que de là tu voies plus commodément autour de toi tout ce qui est dans le monde. Je ne t'ai fait ni céleste, ni terrestre, ni mortel, ni immortel, afin que, comme si tu étais le modeleur et le sculpteur de toimême, maître de toi et ne dépendant que de toi, tu te donnes à toi-même la forme que tu préféreras. Tu pourras dégénérer en devenant un de ces êtres inférieurs que sont les animaux sans raison; tu pourras être regénéré en devenant un de ces êtres supérieurs qui sont divins, au gré de ton esprit. ${ }^{5}$

Pic affirme ici que l'être humain est indéterminé, qu'il n'y a pas à proprement parler de nature humaine, d'humanité, mais que l'homme est en puissance à la fois animal et être divin. Ce n'est pas une pensée révolutionnaire. On lit déjà chez Salluste: "Toute notre force réside dans l'âme et dans le corps; nous utilisons davantage l'âme pour commander, le corps pour servir; l'une nous est commune avec les êtres divins, l'autre avec les bêtes." ${ }^{6}$ Ces thèmes dérivent de Platon; ils sont commun à toute philosophie dualiste.

La deuxième idée dans le texte de Pic est que l'homme choisit lui-même sa place définitive au côté des bêtes ou des êtres divins, sur la terre et au ciel, avec le corps ou avec l'âme. C'est à ce choix en effet que se réduit l'image en apparence grandiose et novatrice de l'homme qui se sculpte lui-même. On reconnaît l'idée de libre-arbitre; cette notion, fondamentale dans la théologie chrétienne, a donné lieu à des débats, à des thèses diverses selon le poids respectif qui est attribuéà la liberté humaine et à la grâce divine. Pour Pic, l'homme décide lui-même de sa destinée, mais s'iln'a besoin de personne pour choir au rang d'animal, ce n'est pas par ses seules forces qu'il s'élève. Dire qu'il “est regénéré" (au passif) signifie à la fois qu'il est dans un état présent dégénéré (son animalité potentielle, sa soumission au corps) et qu'il ne se regénère pas lui-même. ${ }^{7}$ Pic souligne très fortement le rôle du libre-arbitre, mais loin de rompre avec la pensée scolastique, il n'est ici sans doute que l'écho de Duns Scot (ca 1265-1308) dont les doctrines régnaient à la Sorbonne lorsque Pic vint y étudier pendant un an, ou mieux encore peut-être de Henri de Gand $(?-1293){ }^{8}$

Et d'ailleurs doit-on considérer Pic comme un humaniste au sens $\mathrm{n}^{\circ} 1$ ? Se considérait-il lui-même comme tel? Certes il connaît la littérature latine, et la grecque; il a écrit plusieurs poèmes, des discours; mais il n'était pas humanista: il n'a pas commenté de textes littéraires comme son ami Politien; il était en fait philosophe et théologien. Il suffit de considérer quelques-unes de ses oeuvres: 
le De Ente et uno au titre parlant, les Conclusiones DCCCC quas olim Romae disputandas exhibuit. Cette discussion publique de conclusions déposées à l'avance est un exercice scolastique comparable à une soutenance de thèse. Dès les premières lignes, on y lit: "en énonçant ces opinions (des sages Chaldéens, Arabes, Hébreux, Grecs, Égyptiens et Latins) Jean Pic de la Mirandole, comte de la Concorde, n'a pas imité le brillant de la langue romaine, mais la manière de parler des très célèbres disputeurs parisiens parce que la plupart des philosophes de notre temps l'utilisent"; 9 en voici un échantillon: "Species intelligibiles non sunt necessariae, et eas ponere, non est bonis Peripateticis consentaneum." ${ }^{10} \mathrm{Pic}$, par choix délibéré, a donc adopté ici le latin "d'Albert le Grand, de Thomas d'Aquin, de Henri de Gand, de Jean Scot, de Gilles de Rome, de François de Meyronnes et autres péripatéticiens."

Dans son Heptaplus il commente les premiers versets de la Genèse sur la création du monde à l'aide des doctrines de Platon, d'Aristote, d'Hermès Trismégiste, des Oracles chaldaïques, de la théologie chrétienne, de la Kabbale; on y rencontre Averroès, Avicenne et des théologiens juifs à côté des Pères de l'Église et des scolastiques. Ce n'est pas le monde intellectuel des humanistae. D'ailleurs, en son temps, certains d'entre eux reprochèrent à Pic son attachement au latin de la Sorbonne. Parmi eux Ermolao Barbaro, éditeur et commentateur de Pline l'Ancien. Pic résume ainsi le grief qui lui est adressé: "J' aurais perdu, dis-je, dans Thomas, Jean Scot, dans Averroès, mes meilleures années, tant de veilles, pendant lesquelles $\mathrm{j}$ 'aurais pu être quelque chose dans les bonnes lettres." ${ }^{2}$ Barbarolui avaitécrit: "Homo lepidissimus, humanissimus, latinissimus, barbaros contra Barbarum defendis." ${ }^{13}$ La place de "humanus" entre "lepidus" et "latinus" montre qu'il désigne ici une qualité littéraire. "Latinus": Pic sait écrire en bon latin, pur, correct et élégant; "lepidus": il est plaisant, agréable, fin; "humanus": il connaît bien les humanae litterae, la littérature antique, il est cultivé, lettré. Les "barbares" dont Pic avait pris la défense contre leur critique Barbaro sont les universitaires qui écrivent ce latin dont on a vu un échantillon, qui manquent de goût et de culture littéraire.

Pic dans la même lettre dit encore une chose décisive pour la question présente. Les philosophes, explique-t-il, n'avaient pas à étudier la langue latine; leur domaine de recherches était le ciel, les astres, l'univers, les éléments (la physique était alors une partie de la philosophie): "Les philosophes ont séparé la sagesse de l'éloquence; les historiens, les orateurs, les poètes, ce que déplore Philostrate, ont séparé l'éloquence de la sagesse." ${ }^{14}$ Cette exclusion mutuelle est déplorable peut-être, mais la partie la plus importante pour Picn'est pas l'éloquence, la littérature, la langue, c'est la sagesse, la philosophie, 
la pensée. "Nous pouvons vivre sans langue, même si ce n'est peut-être pas facile, mais nous ne pouvons absolument pas vivre sans pensée. Celui qui ne pratique pas la belle littérature n'est pas cultivé (humanus); mais celui qui est étranger à la philosophie n'est pas homme (homo)."15 Très consciemment donc, le prétendu représentant pas excellence de l'humanisme oppose, au lieu de les relier, la culture littéraire, spécialité de l'humanista, à ce que nous donne l'humanitas, et qui est la pensée, la philosophie, la lecture de Platon, d'Aristote, de Jean Scot, d'Avicenne, des scolastiques. Pic rejette expressément le médiocre calembour étymologique que certains utilisaient comme argument pour soutenir que l'étude des litterae humanae nous rend vraiment hommes. ${ }^{16}$ L'antithèse pertinente n'est pas entre anthropocentrisme (qu'on chercherait en vain chez Pic) et théocentrisme, mais entre littérature, domaine du beau style, et philosophie, domaine du vrai. Pic en donne comme exemple, d'une part, le poète Lucrèce qui soutient des aberrations, mais les dit latine et eleganter; d'autre part, le scolastique Jean Scot qui dit la vérité, même si c'est insulse, ruditer, non latinis verbis. ${ }^{17}$ Pic lui-même a les deux cultures: il est capable d'écrire en latin élégant, il est humanus, mais il préfère le latin "barbare" des scolastiques quand il recherche la vérité, en philosophe. ${ }^{18}$

$$
* * *
$$

Il faut généraliser cette antithèse. L'humanisme s'oppose à la scolastique non pas comme une philosophie à une autre, mais comme en d'autres temps les lettres s'opposeront aux sciences. Le conflit n'est pas seulement un débat d'idées; il met en jeu des institutions. Les scolastiques, philosophes et en même temps théologiens, sont des Dominicains et des Franciscains qui, au cours du treizième siècle, se sont introduits dans les universités (à la Sorbonne en particulier, créée au début du siècle), puis s'en sont rendus seuls maîtres et, grisés par Aristote nouvellement découvert, en ont bientôt fait presque l'unique auteur pour toutes les disciplines (droit et médecine étant à part); ils ont exclu les belles-lettres, la littérature païenne, celle d'Ovide ou de Virgile par exemple. C'est contre cette situation que s'insurgent les amoureux de la littérature, les humamistes (généralement des laïcs); mais il leur faudra au moins deux ans pour faire accepter poésie et éloquence à l'université. C'est seulement dans des collèges indépendants de celle-ci qu'ils enseignèrent d'abord.

Mais plutôt que de raconter cette aventure, remontons le temps pour illustrer cette notion de l'humanisme par quelques exemples choisis et traités de manière succincte. On commencera par Valla (1407-1457). Qu'est-ce qui 
fait l'unité de son oeuvre si riche, si variée, si mal connue? ${ }^{19}$ Le point de départ, l'âme, le centre, c'est les Elegantiae Linguae latinae, ouvrage fondamental, l'un des piliers de l'humanisme (sens $\mathrm{n}^{\circ} 1$ ) dans toute la chrétienté latine dès la dernière partie du quinzième siècle. Valla y formule un grand nombre de faits inconnus avant lui concernant la langue latine de l'antiquité qu'il veut restaurer, c'est-à-dire qu'il propose à l'imitation de ceux qui écrivent en latin; il faut prendre ses modèles chez les prosateurs de Cicéron à Quintilien, bannir les mots et constructions usuels à l'université, mais inconnus de ces prosateurs. Qu'est-ce qui a produit cette dégénérescence de la langue que l'on constate chez les scolastiques? Ce sont surtout les écrivains de la fin de l'Antiquité. De Cassiodore, Valla écrit: "Je ne le mentionne jamais parce qu'avec ses rois Théodoric et Alaric, dont il fut le scribe, il parle gothique et barbare." ${ }^{20}$ Boèce suscite encore davantage l'ire de Valla qui proclame son intention de "montrer à ce Latin qu'il ne savait pas parler latin." ${ }^{21}$ Ce n'est pas tant que Boèce ait été contaminé par le parler des Goths, mais il a traduit en latin et commenté une partie considérable de l'oeuvre d'Aristote, en particulier l'Organon (la logique); et les néologismes de cette traduction sont la principale source du vocabulaire scolastique en usage dans les universités, dans les cours et les Sommes théologiques. Boèce et son Aristote latinisé, les philosophes, voilà les ennemis de la belle langue latine et de la littérature.

Dans d'autres oeuvres de Valla, on retrouve la même inspiration polémique, les mêmes adversaires. Son dialogue De libero arbitrio est dirigé lui aussi contre Boèce qui s'était efforcé de concilier philosophiquement le libre-arbitre avec l'omniscience et l'omnipotence divines; Valla s'irrite contre cette prétention de résoudre par la raison un problème qui ne relève pas d'elle, car c'est un mystère de la foi; il faut croire Paul et abandonner Boèce. Valla va jusqu'à mettre en enfer tous les philosophes, avec au premier rang Aristote. ${ }^{22}$ C'est une attaque virulente contre l'inspiration des scolastiques, qui est d'accorder la Révélation avec la raison humaine représentée par le Philosophe. Même hostilité à la philosophie dans un autre dialogue, le De vero bono. Valla montre la contradiction interne ou l'insuffisance, aux yeux mêmes de la raison, des doctrines stoïcienne et épicurienne (qu'il connaît par Cicéron): la première place le souverain bien dans la vertu; la seconde dans le plaisir ou plutôt les plaisirs. Le dialogue entend démontrer contre les uns que le souverain bien réside dans la récompense de la vertu, non dans la vertu elle-même; contre les autres que la vraie voluptas ne consiste pas dans les plaisirs d'ici-bas, mais dans la béatitude de la vie éternelle après la mort; là encore c'est la foi, non la philosophie, qui donne la réponse vraie. 
Ces attaques contre la scolastique sont approfondies d'un autre point de vue dans la Repastinatio dialecticae et philosophiae qui est une critique de la logique d'Aristote. Valla propose de remplacer dans l'enseignement la dialectique par la rhétorique, Aristote par Quintilien; c'est là l'essence de l'humanisme. Ce n'est pas une vision philosophique nouvelle tendant à mettre l'homme à la place de Dieu; ce serait plutôt l'inverse, car les scolastiques, non contents d'employer un latin corrompu, d'avoir exclu l'éloquence et la poésie de la vie universitaire, s'appuient abusivement sur la raison humaine en théologie de même qu'en grammaire où ils préfèrent l'analogieà l'usage. Dans d'autres oeuvres apparaissent d'autres aspects de la même inspiration centrale. Dans ses Annotationes au Nouveau Testament, non seulement Valla critique la Vulgate en philologue, mais il amorce le retour d'une théologie imprégnée d'Aristote à une piété fondée essentiellement sur la lecture du Nouveau Testament, mouvement qui s'épanouira avec Érasme et avec Luther (admirateur du De libero arbitrio). Enfin, dans le De professione religiosorum, Valla s'en prend explicitement aux coupables, les moines, les ordres religieux; il leur reproche de se croire les "pieux" par excellence, comme l'indique le nom qu'ils se font donner de religiosi. On est passé d'une critique de la scolastique à une critique des scolastiques. Valla est dans le camp opposé à celui que défendra Pic de la Mirandole; les mettre ensemble, dans le même mouvement paresseusement nommé "humanisme," c'est s'interdire de comprendre.

Remontons encore et passons du quinzième ou quatorzième siècle, de Valla à Pétrarque (1304-1374). Dans le livre XXIV de ses Epistolae familiares, il adresse des lettres à ceux qu'il considère comme ses vrais contemporains: Cicéron, Virgile, Horace, etc. et même Homère, bien qu'il n'ait pas su le grec. Il reproche à son temps d'ignorer ces grands auteurs, d'être indifférent à la poésie et à l'éloquence. Il reproche aux siècles passés d'avoir laissé se perdre ou se corrompre maints chefs d'oeuvre de l'Antiquité. Il se lamente de vivre dans un medium aevum. ${ }^{23}$ Bien qu'il n' ait pas enseigné, on peut voir en lui, qui redécouvrit les Lettres à Atticus et le Pro Archia, le père fondateur de l'humanisme. Mais le plus intéressant pour notre sujet c'est son long pamphlet De sui ipsius et multorum ignorantia. Quelques jeunes Vénitiens, étudiants à Padoue, avaient reprochéàl'admirateur de Cicéron d'ignorer Aristote. Pétrarque se justifie et contre-attaque, mais il commence par une prière:

Ô Jésus bienfaisant et sauveur, véritable Dieu et dispensateur de toutes les lettres et du talent [ingenii], véritable roi de la gloire et maitre des vertus, c'est toi que j'implore en suppliant, l'âme agenouillée, pour que, si tu ne veux pas me donner davantage, mon lot du moins soit d'être un homme de 
bien: or, si je ne t'aime pas ardemment et ne te vénère pas pieusement, je ne peux pas l'être. ${ }^{24}$

On voit que Pétrarque, loin de vouloir émanciper l'homme, lui donner l'autonomie, fait dépendre du Dieu chrétien non seulement la moralité, mais aussi les lettres et toute l'activité intellectuelle. La conception qui promet à l'homme la vertu sans Dieu est représentée par Aristote, maître des scolastiques; ignorant la Révélation, il ne pouvait savoir ce qu'est la vraie vertu. Pétrarque, s'appuyant sur le De Trinitate d'Augustin, sur Jérôme, "soucieux de ce que dit le Christ et non Aristote," 25 continue sa critique des philosophes: ils s'intéressent moins à la morale qu'à la connaissance de la nature, faisant preuve de cet orgueil que déplore Paul (Rom. 11, 34). Passant ensuite à la connaissance de Dieu que pouvaient avoir les païens, Pétrarque oppose à Aristote Cicéron, son auteur préféré depuis l'adolescence, dont le De Natura deorum du moins est une critique du polythéisme: "Quand il se recueille, par quelles grandes ailes de l'esprit il est soulevé, si bien que parfois on croirait entendre parler non point un philosophe païen, mais un apôtre." ${ }^{26}$ On a ici une esquisse du célèbre dialogue d'Érasme (1467-1536) où l'un des personnages s'écrie: "Saint Socrate, priez pour nous"27; loin que les auteurs païens soient utilisés pour affirmer même timidement un anthropocentrisme, ils sont enrôlés au service de la piété chrétienne.

Pétrarque critique enfin le style d'Aristote (qu'il ne connaît qu'en traduction); il ne s'agit pas de beauté, mais:

J'ai lu, sauf erreur, tous les ouvrages de morale d'Aristote, j'ai entendu des cours sur quelques-uns et, avant qu'on ait mis à nu mon énorme ignorance, j'avais cru comprendre quelque chose et peut-être quelquefois grâce à eux je suis revenu à moi plus savant, mais non pas, comme il aurait fallu, meilleur et je me suis plaint souvent à moi-même, quelquefois à d'autres, que de la sorte n'était pas accompli ce qu' au livre I de l'Éthique le même philosophe a dit en préface: que l'on étudie cette partie de la philosophie non point pour savoir, mais pour devenir meilleur. Car je vois bien qu'il définit et subdivise remarquablement la vertu, qu'il traite avec pénétration de ce qui est propre à chaque vice ou vertu. Après avoir étudié cela, je suis un peu plus savant que je ne l'étais; mais mon âme est inchangée, ma volonté inchangée, moi-même inchangé. Car c'est une chose de savoir, et une autre d'aimer, une chose de comprendre, et une autre de vouloir. Aristote enseigne, je ne le conteste pas, ce qu'est la vertu; mais les aiguillons et les ardeurs du verbe, qui stimulent et enflamment l'esprit à l'amour de la vertu et à la haine du vice, sa manière de s'exprimer [lectio] ne les possède pas ou ne les possède qu'en très petit nombre. Celui qui les cherche les trouvera chez les nôtres [les écrivains 
latins], surtout Cicéron et Sénèque et, ce qui pourra étonner, chez Horace, poète au style certes raboteux, mais dont la pensée est très agréable. À quoi servira de savoir ce qu'est la vertu, si quand on la connaît on ne l'aime pas? À quoi la connaissance du péché est-elle utile, si une fois connu on ne le prend pas en horreur? ${ }^{28}$

Pétrarque utilise ici la distinction fondamentale en rhétorique: docere, delectare, mouere; admirable peut-être pour la première fonction, Aristote est nul pour les deux autres: il n'a point d'agrément, et surtout il n'émeut pas, il ne touche pas la sensibilité, il n'incite pas à rechercher le bien et à fuir le mal, il ne change pas les dispositions de ses lecteurs. Ce grief sera repris par Érasme contre les docteurs de la Sorbonne:

je ne puis lire les ouvrages de Cicéron Sur la vieillesse, Sur l'amitié, Sur les devoirs, Sur les questions tusculanes, sans couvrir quelquefois le livre de baisers et sans être saisi de vénération pour cette âme sainte inspirée par le génie du ciel. À l'inverse, quand je lis certains livres récents sur la politique, l'économie ou l'éthique, ô Dieu immortel! comme ils sont froids en comparaison des anciens! bien plus, comme ils semblent ne rien sentir de ce qu'ils écrivent! à tel point que pour ma part je préférerais voir périr tout Scot [admiré, on s'en souvient, par Pic] avec plusieurs de ses pareils plutôt que les livres du seul Cicéron ou du seul Plutarque; ce n'est pas que je condamne ceux-là en bloc, mais je sens que ceux-ci me rendent meilleur, tandis qu'après avoir lu les premiers j'ai, sans savoir pourquoi, le coeur plus froid envers la véritable vertu et plus enclin à la polémique. ${ }^{29}$

La vertu dont parle Pétrarque ou Érasme est une vertu chrétienne. L'auteur du Secretum qui, pour confesser ses péchés - amour charnel et amour de la gloire - choisit la forme d'un dialogue avec Augustin, l'éditeur, traducteur et commentateur du Nouveau Testament, s'opposent aux scolastiques non point en raison d'une philosophie qui donnerait une place plus grandiose à l'homme, mais parce qu'ils veulent que l'écrivain sache flectere (ou mouere). L'humanisme n'est pas seulement l'amour des langues antiques, c'est l'amour de l'éloquence; et lire Cicéron ou Virgile, Sénèque ou Terence, c'est le meilleur moyen d'apprendre à être éloquent.$^{30}$ Quand les humanistes disent que l'homme se définit par le langage (et non par la raison), il faut le comprendre dans le même sens: parler ne se réduit pas à argumenter, c'est aussi plaire et surtout toucher le coeur, éveiller des passions, amener ceux à qui l'on s'adresse à se transformer intérieurement. Les humanistes écrivent des discours, des déclamations, des lettres, des dialogues; dans tous ces genres l'auteur accomode son langage à l'autre afin de l'émouvoir. Le scolastique, l'auteur de 
Sommes, Conclusions ou Disputations, ne vise qu'à démontrer, définir, distinguer et diviser, argumenter et réfuter; il ne s'adresse qu'à l'intellect, il est impersonnel et froid.

\section{***}

Bref, du quatorzième au seizième siècle, l'humanisme ne saurait être défini comme une exaltation de "l'humanité de l'homme" ou même comme l'esquisse ou l'annonce d'une telle position. Cette conception n'est qu'un jeu de mots, un exemple éclatant des méfaits de l'étymologie érigée en preuve. ${ }^{31}$ Voir en Pétrarque, en Valla, en Politien, en Érasme, en Budé, les tenants ou les précurseurs d'une pensée qui tendrait à mettre l'homme à la place de Dieu est une aberration. Si au seizième siècle certains font un pas dans la direction d'un anthropocentrisme, ce sont des philosophes, et non des humanistes; je ne sais si Pomponazzi peut être classé parmi ces novateurs, mais quand on lit sous sa plume: inter sceleratos versatur adeoquod nunquam vidit aliquem virum bonum, ${ }^{32}$ on ne peut qu'approuver Margolin qui se refuse à voir en lui un humaniste. ${ }^{33}$

Ce qui doit être rappelé au terme de ces analyses rapides, c'est qu'au treizième siècle les nouveaux ordres monastiques, dominicains et franciscains, devenus en quelques décennies maîtres des universités, y firent régner Aristote, en exclurent les belles-lettres. Thomas d'Aquin classe la poésie au dernier rang des arts; d'autres dénoncent les poèmes qui chantent les dieux païens et s'opposent à ce qu'ils soient étudiés ${ }^{34}$; contre eux, en partie pour justifier la lecture de Virgile, d'Ovide, d'Homère, de Stace, etc. Boccace écrira sa Genealogiadeorum gentilium. Il serait absurde d'en conclure que les humanistes avaient quelque inclination pour le polythéisme, quoi qu'ait pu en penser le pape Adrien VI qui, au début du seizième siècle, voyait dans les poètes des païens. ${ }^{35} \mathrm{Si}$ Dominicains et Franciscains étaient allés au bout de leur rêve, sans doute auraient-ils au nom de la vérité fait disparaître encore plus d'oeuvres que n'en avaient détruites les autorités ecclésiastiques huit siècles plus tôt, lorsqu'elles anéantirent certains livres d'Origène. Ils essayèrent d'empêcher l'expansion du grec, "source d'hérésies." Mais du ressentiment des humanistes contre les scolastiques, contre les moines, il est aberrant de conclure qu'ils n'étaient plus vraiment chrétiens, qu'ils tendaient à mettre l'homme à la place de Dieu. Au contraire, c'est d'un point de vue chrétien que Pétrarque, Valla et bien d'autres s'en prennent aux scolastiques, leur reprochant de préférer Aristote, ce païen, à l'Écriture, la raison humaine à la Révélation, un homme 
à Dieu; à l'accusation portée contre le grec, ils rétorquent par des formules de Jérôme, disant que la source principale des hérésies est la philosophie! Bref, il faut pouréviter la confusion dans les pensées dépouiller la notion d'humanisme des oripeaux philosophiques dont on a voulu la parer et s'en tenir au sens précis, restreint, du mot: "mouvement né avec Pétrarque qui exalte l'étude et l'imitation des poètes, orateurs et historiens antiques et s'insurge contre le despotisme intellectuel qu'exercent dans les universités et ailleurs les moines fanatiques d'Aristote et pourtant ennemis de la littérature païenne comme dangereuse pour la foi chrétienne."

L'admiration des humanistes pour les auteurs anciens n'a pas de suite philosophique, elle ne leur donne pas une conception nouvelle de l'homme, mais elle a une conséquence religieuse considérable: puisque des êtres tels que Socrate ou Cicéron ont pu avoir une vie, une pensée, une morale si parfaitement conformes à l'enseignement du Christ, il serait inconcevable qu'ils ne soient pas sauvés. Le personnage du Convivium religiosum parle des âmes saintes de Virgile et d'Horace; ils étaient saints sans appartenir à l'Église, chrétiens sans avoir reçu le baptême, sans avoir entendu parler du Christ; ou pour le dire autrement, il convient de distinguer deux sens du mot chrétien, deux sens du mot Église:l'un institutionnel, corporel, l'autre intérieur et spirituel, le seul qui importe vraiment. Hors de l'Église point de salut: mais ce n'est pas l'Église des prêtres, des sacrements, du pontife romain, c'est l'Église du Christ dont les membres sont connus de Dieu seul. "Hors de l'Église point de salut" devient un pléonasme, puisque la véritable Église est constituée de ceux qui sont sauvés. Cette conception dont Pétrarque s'approche quand il parle de Cicéron est formulée en toute clarté par Érasme, dont elle fait la grandeur.

Une périodisation, comme on dit de façon barbare (mais je ne vois pas d'autre mot), devrait, en ce qui concerne l'histoire des idées en Europe, ${ }^{36}$ renoncer au concept inconsistant de Moyen Âge, considérer la révolution scolastique du treizième siècle comme l'événement le plus important par ses côtés négatifs aussi bien que positifs; l'humanisme est une lente et difficile revanche contre cette main-mise qu'il faut bien dire totalitaire ${ }^{37}$ des ordres religieux sur l'université, dont une conséquence avait été l'éviction des belleslettres par la philosophie, des poètes par Aristote. La revanche ne fut complète que trois siècles plus tard quand fut créé l'enseignement des Jésuites: cela signifiait que les autorités catholiques reconnaissaient enfin la légitimité d'étudier la littérature païenne et même de faire reposer sur elle toute la formation, sans que cela mîten danger la foi. Quant à la conception spiritualisée de l'appartenance à l'Église, ne la retrouverait-on pas aussi d'une certaine 
manière deux siècles plus tard dans l'attitude des Jésuites à l'égard des croyances chinoises? Quoi qu'il en soit, l'humanisme, après avoir absorbé son ancien adversaire, puisque la philosophie fait partie des études littéraires, a duré jusqu'à nos jours. Il est maintenant moribond, mais ceci est une autre histoire.

\section{Université de Paris IV - Sorbonne}

\section{Notes}

Cet article a d'abord paru dans les Annales Latini Montium Arvernorum, le bulletin du Groupe d'études latines de l'Université de Clermont-Ferrand ( $\left.{ }^{\circ} 23,1996,87-101\right)$. Sa forme est légèrement remaniée ici.

1. “... Hélas, j'en ai tant connu [d'humanistes]! L'humaniste radical (...). L'humaniste dit 'de gauche' (. . .). L'écrivain communiste (. . .). L'humaniste catholique (. . .). Ça, ce sont les grands premiers rôles. Mais il y en a d'autres: le philosophe humaniste, qui se penche sur ses frères comme un frère aîné et qui a le sens de ses responsabilités; l'humaniste qui aime les hommes tels qu'ils sont, celui qui les aime tels qu'ils devraient être (. . .), l'humaniste joyeux qui a toujours le mot pour rire, l'humaniste sombre, qu'on rencontre souvent aux veillées funèbres" (La nausée, éd. 1950, p. 149). Sartre prend ici le mot dans une acception proche de celle qui sera définie ci-dessous comme $n^{\circ} 3$. Après guerre, il prendra le mot "humanisme" - au sens $\mathrm{n}^{\circ} 2$ - à son compte.

2. Jean-Claude Margolin, L'humanisme en Europe au temps de la Renaissance (Paris, Presses Universitaires de France, Coll. Que sais-je?, 1981).

3. Après les articles de Billanovich voir celui de Rino Avesani, "La professione dell' umanista nel Cinquecento," Italia Medioevale e Umanistica, 13 (1970), 205-232. Parmi les nombreuses citations qui y figurent, voici un extrait de la Vita Latina de Savonarole, due à un frère anonyme de S. Marco (1528): "Dominus Bartholomeus Fontius, magnus humanista, in tantum se convertit, quod, relictis poetis [c'est-à-dire les auteurs païens], in publico ginnasio et Hieronymi epistolas ac B. Ambrosium De Officiis publice legebat" (p. 208). Dans les établissements de la Société de Jésus les humanistae sont les élèves de rang inférieur aux rhetores et supérieurs aux grammatici (p. 227). À la veille de la dernière guerre, les lycéens français ornaient quelquefois le revers de leur veste d'insignes en métal doré: un $H$, un $\rho$, un $\phi$ selon la classe: seconde (humanistes), première (rhétoriciens), philosophie; de la $6^{\mathrm{e}}$ à la $3^{\mathrm{e}} \mathrm{c}^{\prime}$ était les classes de grammaire. Pour humanitas pris en ce sens voir ci-dessous les texte de Valla, note 21 .

4. L'une des oeuvres importantes de l'humanisme en Italie au quinzième siècle est la Cornucopia de Perotti, commentaire des Épigrammes de Martial.

5. Aulu-Gelle XIII, XVII, explique que humanitas est en général l'equivalent du grec philanthropia, et signifie benivolentiam erga omnes homines promiscam; mais certains, dit-

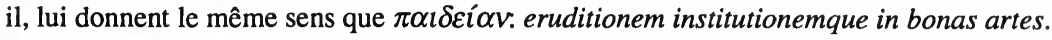
Dès l'antiquité le même mot a les acceptions n $n^{\circ} 1$ et n ${ }^{\circ} 3 d^{\prime}$ 'humanisme. Dans la correspondance de Politien humanitas et humanus ont le plus souvent le sens 3. 
6. "Nec certam sedem, nec propriam faciem [. . . ex tui animi sententia regenerari" (De hominis dignitate). On sait que ce titre n'est pas de Pic, mais des éditeurs de ses oeuvres complètes en 1550. Le titre authentique était: Oratio quaedam elegantissima; ce dernier mot signifie que Pic y a adopté le style humanus et non pas parisien.

7. "Nostra vis in animo et corpore sita est; animi imperio, corporis seruitio magis vtimur; alterum nobis cum dis, alterum cum beluis commune est" (De coni. Cat., I, 2).

8. Pour ce verbe, voir 1 Pet. 1,3: "Benedictus Deus [...] qui secondum magnam misericordiam suam regenerauit nos in spem viuam." Louis Valcke insiste avec raison sur la forme passive dans "Jean Pic de la Mirandole (1463-1494): quatre ouvrages récents," Dialogue, XXIV (1995), p. 346. Cf. H. de Lubac, Pic de la Mirandole (Paris, Aubier-Montaigne, 1974), pp. 116-129.

9. Tous deux figurent dans les Conclusiones. Dans sa Lectura in librum secundum sententiarum II dist. 25 Q. unica: De causa effectiva actus volendi Scot rejette les deux opinions extrêmes qui donnent pour cause à l'acte de volonté soit l'objet seul soit la volonté seule ( $=$ Henri de Gand) (Opera Omnia, t. XIX, Cité du Vatican, 1993, p. 253). Voir Gilson, Gandillac (Encyclopaedia Universalis), Chevalier (Histoire de la pensée).

10. "De adscriptis . . placitis disputabit publice Ioannes Picus Mirandulanus, Concordia comes; in quibus recitandis non Romanae linguae nitorem, sed celebratissimorum Parisiensium disputatorum dicendi genus est imitatus, propterea quod eo nostri temporis philosophi plerique omnes vtuntur" (Conclusiones DCCCC). La "disputatio" est un acte universitaire qui confronte les tenants de thèses différentes.

11. C'est la première des 900 Conclusions, elle est secundum Albertum: "Les espèces intelligibles ne sont pas nécessaires, et les poser ne s'accorde pas avec la pensée des bons péripatéticiens."

12. Énumération précédant les Conclusiones. Jean Scot est Duns Scot.

13. "Perdiderim ego, inquam, apud Thomam, Ioannem Scotum, apud Averroem, apud Albertum meliores annos, tantas vigilias, quibus potuerim in bonis litteris fortasse nonnihil esse!" (Lettre à Barbaro in Riccardo Ricciardi, La letteratura italiana. Storia e testi, vol. 13, Prosatori latini del Quattrocento par Eugenio Garin [Milan \& Naples, 1952], p. 806). La lettre est de 1485. "Tantas" a le sens de "tot," comme souvent alors.

14. Humanissimus est chez Aulu-Gelle, loc. cit.: "[bonas artes] qui sinceriter percupiunt adpetuntque, hi sunt vel maxime humanissimi."

15. "Abiunxerunt philosophi sapientiam ab eloquentia; abiunxerunt historici, rhetores, poetas, quod deflet Philostratus, eloquentiam a sapientia" (Lettre à Barbaro, p. 820).

16. "Viuere sine lingua possumus, forte non commode; sed sine corde nullo modo possumus. Non est humanus qui sit insolens politioris litteraturae; non est homo qui sit expers philosophiae" (Lettre à Barbaro, p. 820).

17. Voir Jean Paulhan, Alain, ou la preuve parl'étymologie, in Oeuvres complètes, t. III (Paris, Au Cercle du Livre Précieux), p. 261 sq.

18. Ibid., p. 822. Duns Scot est choisi parce que (redisons-le) Pic a suivi des cours à la Sorbonne où régnaient les scotistes, honnis quelques années plus tard par Érasme. 
19. L'oeuvre de loin la plus considérable de Pic relève de l'histoire de la science: c'est une réfutation en XII livres de l'astrologie, Disputationes in Astrologiam, dédiée aux "veritatis amatoribus"; la langue en est assez technique pour n'être pas considérée comme humana, sans que pourtant elle soit "scolastique."

20. L'un le voit occupé à rétablir le véritable Aristote, l'autre fait de lui un "puriste."

21. "De ... Cassiodoro qui apud nonnullos in pretio est, nunquam ides facio mentionem, quia cum regibus suis Theodorico et Alarico, quorum scriba fuit, Gotthicum sonat et barbarum" (Elegantiae, IV, p. 88). Dans la Préface au Livre III des Elegantiae Valla déplore que les jurisconsultes emploient un latin "gothique": "neque hoc dico vt iuris studiososcarpam, imo vt adhorter potius persuadeamque sine studiis humanitatis non posse quam cupiunt assequi facultatem"; pour la même raison il critique ensuite les philosophes "dont les livres ne seraient même pas compris par les Goths et les Vandales," les professeurs de grammaire et ceux de rhétorique (les artistae).

22. "Huic homini Romano ostendam Romane loqui nescire" (Elegantiae, VI, 35).

23. De libero arbitrio, éd. Jacques Chomarat (Paris, Vrin, 1983), pp. 47-49, 99-108; tous les philosophes sont en Enfer; "Quorum in primis fuit Aristoteles, in quo deus optimus maximus superbiam ac temeritatem cum ipsius Aristotelis, tum ceterorum philosophorum patefecit atque ideo damnauit" et "Religio christiana non probatione nititur, sed persuasione quae praestantior est quam probatio" (Elegantiae, V, 30).

24. J. Chomarat, "Érasme et le Moyen-Âge," Mots et coyances, sous la direction de Jacques Chomarat (Genève, Droz, 1995), p. [33].

25. "Et, o alme salutiferque Ihesu, vere literarum omnium et ingenii Deus ac largitor, vere rex glorie ac virtutum domine, te nuncflexisn anime genibus supplexoro, vt si michinon amplius vis largiri, hec saltem portio mea sit, vt vir bonus sim; quod, nisi te valde amempieque colam, esse non possum" (De sui ipsius et multorum ignorantia, in La letteratura italiana, vol. 7, Petrarca [Milan \& Naples, 1955],p. 714); Pier Giorgio Ricci traduit: "re della gloria verace," mais il paraît préférable de voir en "vere" une épithète de "rex" (comme le premier "vere" l'était de "Deus") plutôt qu'un équivalent de "verae."

26. Ibid., p. 722. Le texte de Jérôme dit "Paul," et non "le Christ."

27. "His ipsis in libris, quod de diis, non de deo scribit, vbu sese colligit, quantis ingenii alis attollitur, vt interdum non paganum philosophum, sed apostulum loqui putes," Ibid., p. 728.

28. Convivium religiosum, Opera Omnia ASD, I-3, 254, 710 (Ce texte est de 1522).

29. "Omnes morales ... non horretur," Op. cit., pp. 744-746.

30. Convivium religiosum, 251, 621-630. Érasme s'est-il inspiré de Pétrarque?

31. Les religieux critiquaient la lecture des libri saeculares en rappelant l'accusation portée contre Jérôme: "Ciceronianus es, non Christianus" (Epist. 22, 30); "Sed cum Hieronymus quod Ciceronianus est repraehenditur, id repraehenditur quod studiosus eloquentiae esset. Ideoque damnati et repulsi intelliguntur, qui comparandae eloquentiae gratia lectitantur" (Valla, Eleg., IV, Praefatio). 
32. On "démontrerait" de même que la police veut policer les hommes. Cf. note 16.

33. Pomponazzi, De Fato, I, III, 26, éd. Lemay (Lugano, Thesaurus Mundi, 1957).

34. J.- C. Margolin, Op. cit., p. 12.

35. Par exemple le Dominicain Dominici dans sa Lucula noctis (1405).

36. Paolo Giovio, Vita Hadriani Sexti, p. 144, cité par Haig Gaisser, Catullus and his Renaissance Readers (Oxford, Clarendon Press, 1993), p. 352, note 125.

37. On considère habituellement comme caractéristique de la "nouvelle vision du monde" apparue au seizième siècle le système de Copernic; du treizième au quinzième siècle, l'homme était au centre du monde (encore chez Pic, voir n. 6: "Medium te mundi posui"); la pensée moderne lui ôte donc ce privilège, encore plus qu'elle reconnaît l'étendue illimitée de l'univers. Cela va évidemment à l'encontre de la prétendue visée de l'humanisme: faire "de l'homme le centre et le maître du monde."

38. Les Dominicains en tout cas avaient été créés pour faire la chasse à tout ce qui pouvait sembler une menace contre l'orthodoxie. Sur le retour des belles-lettres voir Étienne Gilson, La philosophie au Moyen Âge, vol. 2 (Paris, Payot, 1962), ch. X. On trouvera une critique de la notion de Moyen Âge dans l'ouvrage signalé, note 24. Je remercie vivement $\mathrm{L}$. Kolakowski d'avoir attiré sur lui l'attention (Times Literary Supplement, 1 déc. 1995). 